\title{
Electric Field Induced Resistance Change in PEDOT-PSS Films
}

\author{
S. Bindu ${ }^{{ }^{*}}$ and M. S. Suresh ${ }^{1}$ \\ ${ }^{1}$ Department of Electronics and Communication, B.N.M. Institute of Technology, India.
}

Authors' contributions

This work was carried out by author SB under the guidance of author MSS. All the design calculations and experimentations were done by author SB along with literature survey. The paper draft was prepared by her and the second author reviewed it for details, accuracy and theoretical aspects. Both authors read and approved the final manuscript and agree to the terms of publication.

\begin{abstract}
Article Information
DOI: 10.9734/BJAST/2017/33159

Editor(s):

(1) Luigi Maxmilian Caligiuri, Faculty of Science, University of Calabria, Italy and Foundation of Physics Research Center (Director)- FoPRC, Italy.

Reviewers:

(1) A. Ayeshamariam, Khadir Mohideen College, Adirampattinam, India. (2) Kasem K. Kasem, Indiana University Kokomo, Indiana. Complete Peer review History: http://www.sciencedomain.org/review-history/18997
\end{abstract}

Original Research Article

Received $31^{\text {st }}$ March 2017

Accepted $29^{\text {th }}$ April 2017

Published $10^{\text {th }}$ May 2017

\begin{abstract}
Resistance of PEDOT-PSS films changes considerably with time after application of DC voltage. Since, variation of resistance over time can lead to serious errors when used as a strain gauge or gas sensors or any device, a detailed study of the variation of film resistance with time has been made. It has been observed that $A C$ resistance is stable with time (constant) compared to DC resistance which has been explained in terms of contact diode model. It has also been found that doping with graphite leads to lower variation (better stabilization) in the resistance over time. The results indicate that the variation of resistance is an interfacial phenomenon at the contacts and either an ohmic contact or AC measurement removes this behavior. The change of resistance is attributable to high electric field in the interfacial region of the PEDOT-PSS/metal contact.
\end{abstract}

Keywords: Conducting polymers; PEDOT-PSS; rectifying contacts; ohmic contacts; depletion region; electric field. 


\section{INTRODUCTION}

The resistance of conductive films is normally constant on application of DC voltage or electric field. A small change is expected due to joule heating of the film due to power dissipation and consequent increase in local temperature [1]. The change in resistance depends on the temperature co-efficient of resistance which is normally negative and the resistance of film reduces. When we applied DC voltage to PEDOT-PSS films to measure its resistance we found that the film resistance increased with time, contrary to expectations. The resistance measurements were made as part of building high gauge factor strain gauges using PEDOTPSS films and the constancy of resistance for changes other than strain is an important parameter to build strain gauges. Many have reported about strain gauges built from PEDOTPSS films [2-3] but none have reported instability of film resistance on application of DC voltage or the effect of electrical contact material over PEDOT-PSS. It should be recognized here that PEDOT-PSS is a $p$ type semiconductor and the interface between the metal contact and the film may not be ohmic. This paper presents the measurement of resistance of PEDOT-PSS films over time with copper contacts and proposes a model that explains the observations. The study includes measurements on pristine and graphite doped PEDOT-PSS films.

\section{EXPERIMENTAL DETAILS}

PEDOT-PSS films (Pristine and doped) are cast on epoxy glass substrates of dimension $75 \mathrm{~mm}$ * $25 \mathrm{~mm}$ and thickness $0.8 \mathrm{~mm}$. Two copper contacts $10 \mu \mathrm{m}$ thick, $0.5 \mathrm{~mm}$ wide, spaced $1 \mathrm{~mm}$ apart are pre-etched on the substrate to serve as electrical contacts. PEDOT-PSS films $5 \mathrm{~mm} \times 5 \mathrm{~mm}$ are formed overlapping the copper contacts by evenly spreading (drop casting) a known quantity $(20 \mu \mathrm{L})$ of aqueous suspension of PEDOT-PSS (VP AL 4083) procured from H C Stark Germany as shown in Fig. 1. The film is baked in a temperature controlled box for one hour at $45^{\circ} \mathrm{C} \pm 0.1^{\circ} \mathrm{C}$ and the films thus formed had a thickness of $10 \mu \mathrm{m}$, measured using a mitotoyo dial gauge of $1 \mu \mathrm{m}$ resolution. The films formed are encapsulated using flexible transfer adhesive tape to prevent exposure to atmosphere. As will be explained later, the copper contacts on some samples are treated appropriately to make PEDOT-PSS metal contacts ohmic. As the contacts are spaced
$1 \mathrm{~mm}$ apart the effective film area is $5 \mathrm{~mm}^{2}$. Measurements are also made on films with contact width of $1 \mathrm{~mm}$ to study the effect of interfacial area on the resistance. The film resistance is measured by applying voltage, DC or AC, to a potential divider network with the film forming one resistor and another fixed resistor is connected in series to measure the current. Voltages of $1 \mathrm{~V}, 3 \mathrm{~V}, 6 \mathrm{~V}, 12 \mathrm{~V}$ and $24 \mathrm{~V}$ are connected and the resistances of the films are measured over time up to 45 minutes to see the variation of resistance with time after application of voltage.

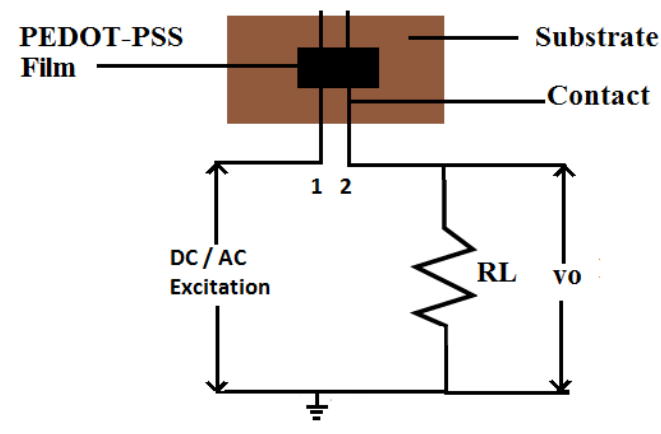

Fig. 1. Circuit used to measure film resistance/impedance

\section{RESULTS AND DISCUSSION}

\subsection{Variation of Resistance of Pristine PEDOT-PSS Films with Time}

Variation of film resistance with time for pristine PEDOT-PSS film for applied DC voltages $1 \mathrm{~V}, 3 \mathrm{~V}$, $6 \mathrm{~V}, 12 \mathrm{~V}$ and $24 \mathrm{~V}$ is shown in Fig. 2. The voltages shown on the figure are the total applied voltage and the actual voltage across the film is $0.7 \mathrm{~V}$, $2.4 \mathrm{~V}, 5.2 \mathrm{~V}, 9.8$ and $19.4 \mathrm{~V}$ respectively. It may be observed that the film resistance at $1 \mathrm{~V}$ is nearly constant with time, slightly reducing with time in fact, while at higher voltages the film resistance increases with time by about 1.3 to 1.6 times the initial value in 45 minutes; higher the voltage higher the variation. At $24 \mathrm{~V}$ change in resistance with time suddenly decreases and is about the same as that at $3 \mathrm{~V}$. At $12 \mathrm{~V}$ there is a small fall in resistance after initial increase.

Fig. 3 shows the initial resistance and resistance after 45 minutes for different voltages. It may be seen that initial resistance is nearly same for all applied voltages while the resistance after 45 minutes peaks around $8 \mathrm{~V}$. 


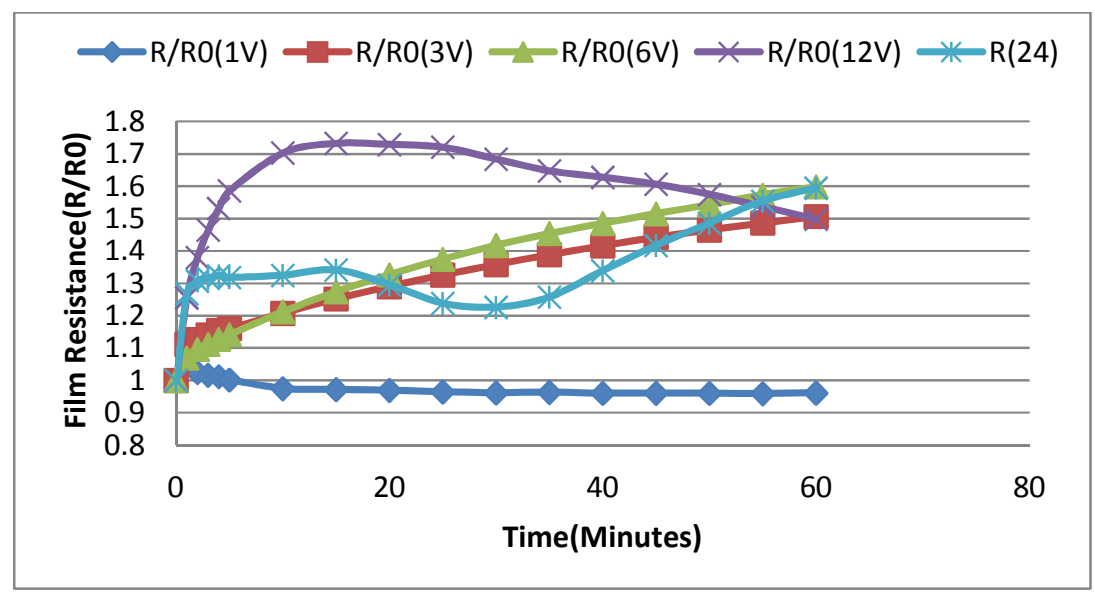

Fig. 2. Variation of film resistance vs. time for pristine PEDOT-PSS film making contact to $0.5 \mathrm{~mm}$ wide copper contact

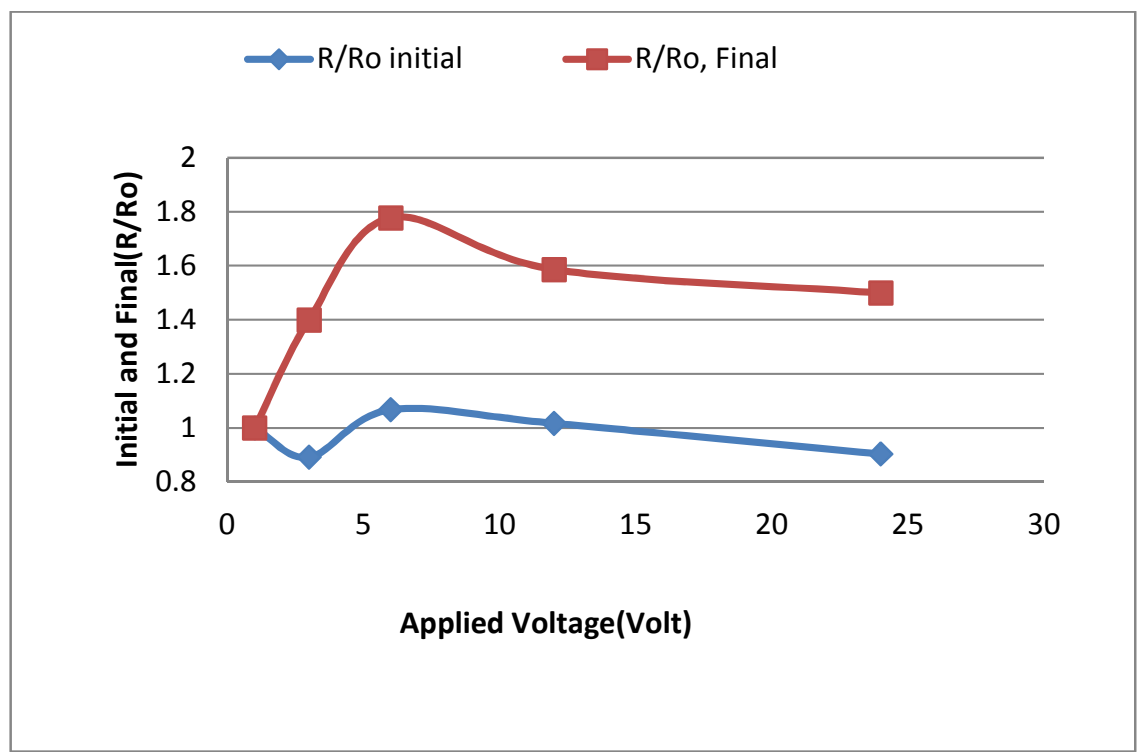

Fig. 3. Variation of initial and final resistances of pristine PEDOT-PSS film for different applied voltages for a period of $\mathbf{4 5}$ minutes

It is reported in the literature that PEDOT-PSS resistance has a negative co-efficient of temperature [4] and is given by;

$$
\alpha_{\mathrm{t}}=-3.5 * 10^{-3} \mathrm{~K}^{-1}
$$

The Joule heating should have reduced the resistance instead of increasing as temperature co-efficient is negative. This clearly indicates that the cause for the observed behavior of film resistance is not joule heating.

A metal semiconductor interface will form either a rectifying junction or an ohmic junction depending on the work functions of the semiconductor film and the contact metal [5-8]. When the interface is rectifying, the total resistance consists of two parts, the leakage resistance of a reverse biased diode and the bulk resistance of the film. An equivalent circuit of such a situation is given by Fig. 4 which presents a reverse biased diode in either direction of current flow together with bulk resistance. Resistance of forward biased diode can be neglected and hence, the total resistance is dominated by the interfacial resistance due to reverse biased metal semiconductor film diode. The presence of diode has been shown in an 
earlier work and the leakage resistance of the diode could be about 5 times or more than the bulk resistance [9].

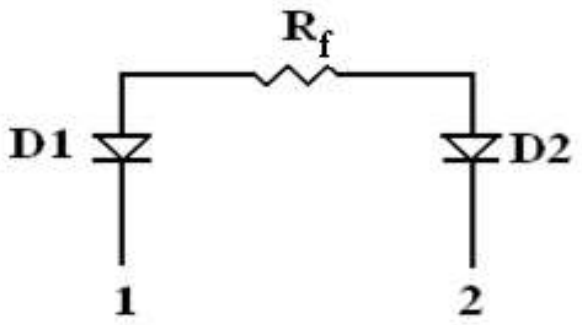

Fig. 4. Model of a polymer film with two metallic contacts

To study the effect of interfacial resistance of PEDOT-PSS film with copper contacts another film is cast with double the copper contact area; i.e the width of copper contact is increased from $0.5 \mathrm{~mm}$ to $1 \mathrm{~mm}$, with the distance between contacts held constant at $1 \mathrm{~mm}$. All other process parameters are held constant.

The variation of PEDOT-PSS film resistance with time for $1 \mathrm{~mm}$ wide copper contacts is shown in Fig. 5. It may be seen that the film resistance increases over a period of 45 minutes by about 4.2 to 4.6 times the initial value compared to about 1.3 to 1.6 times for $0.5 \mathrm{~mm}$ wide copper contacts.

\subsection{Impedance (Resistance) of Pristine PEDOT-PSS Film with AC Excitation}

Invoking the diode model explained earlier, the reverse biased contact diode can be modeled as leakage resistance in parallel with depletion capacitance as shown in Fig. 6. The reverse leakage resistance is bypassed by low impedance of depletion capacitor at sufficiently high frequency. Hence, the measured resistance is that of the bulk of the film if the applied voltage is $A C$ of sufficiently high frequency.

The variation of resistance of pristine PEDOTPSS film measured by applying AC voltage at a fixed frequency of $10 \mathrm{KHz}$ is shown in Fig. 7. It may be observed that the film resistance for an applied AC voltage of $3 \mathrm{~V}(\mathrm{rms})$ and $6 \mathrm{~V}(\mathrm{rms})$ is nearly constant with time.

The above observations prove that the Variation of film resistance with time on application of DC voltage is largely confined to the depletion or the interfacial region and not the bulk.

\subsection{Effect of Graphite Doping on PEDOT- PSS Film Resistance}

A pristine PEDOT-PSS film produces high resistance films and it is common practice to dope PEDOT-PSS to produce films of lower resistance. So, a set of measurements are made on graphite dispersed PEDOT-PSS films with varying graphite concentrations in weight percentages of $0.1 \%, 0.25 \%, 0.5 \%, 0.75 \%$ and $1 \%$. All dimensions of substrate and film are maintained same as earlier.

Fig. 8 shows the variation of film resistance with different concentration of graphite. The resistance is nearly constant at about $1 \mathrm{M} \Omega$ up to $0.5 \%$ and suddenly reduces to $650 \Omega$.

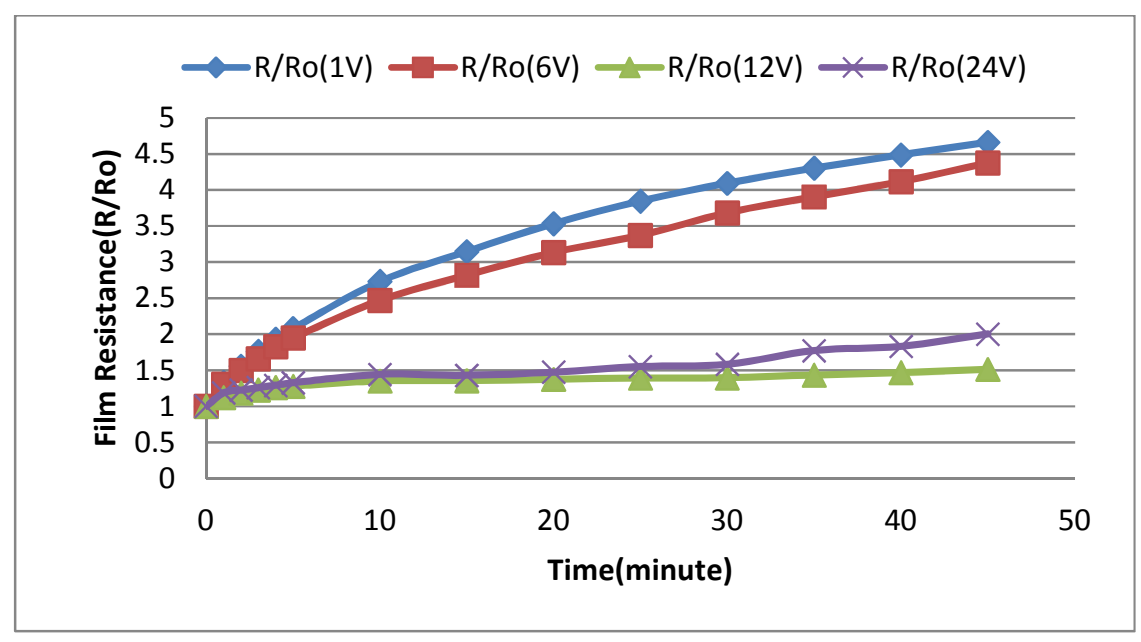

Fig. 5. Variation of film resistance vs. time for pristine PEDOT-PSS film making contact to1mm wide copper contacts 
This behavior is in conformity with the predictions by percolation theory. The general proposition of the percolation theory comes from the fact that the conductivity of a composite dramatically increases at a certain concentration called the percolation threshold of the (filler) material [1011]. At doping/dispersing concentrations above the critical level, the conductivity reaches a plateau and does not rise significantly with further addition of filler. At low graphite loading, the particles are homogeneously distributed without contact between adjacent filler particles and the conductivity of this composite is comparable to that of the pristine material. With increasing graphite concentration, however, clusters of filler particles begin to form and in these clusters the filler particles are in contact with each other. At a certain critical graphite concentration, the growing clusters reach a size which enables a contact between them; a continuous network structure of the conducting filler is formed. This network formation can be detected by a drastic decrease in the electrical resistivity of the composite. Increase in number of parallel paths does not significantly change the conductivity.

\subsection{Measurements on PEDOT-PSS Films Dispersed with Graphite Powder}

Fig. 9 shows the variation of film resistance with time for different concentrations of graphite powder dispersed in PEDOT-PSS for an applied DC voltage of $3 \mathrm{~V}$. Since the resistance of film changes considerably with concentration of dispersed graphite, the resistance values are normalized to their initial values and plotted to get better comparison between films.

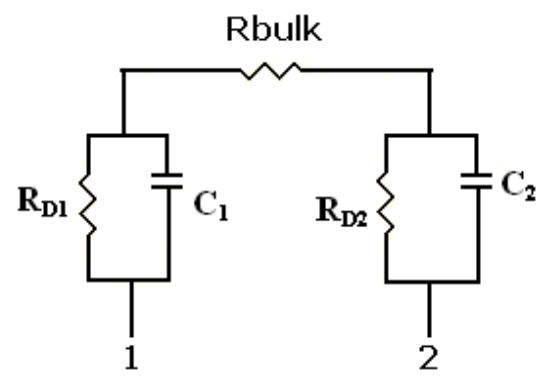

Fig. 6. AC equivalent circuit of contact diode at metal-polymer interface

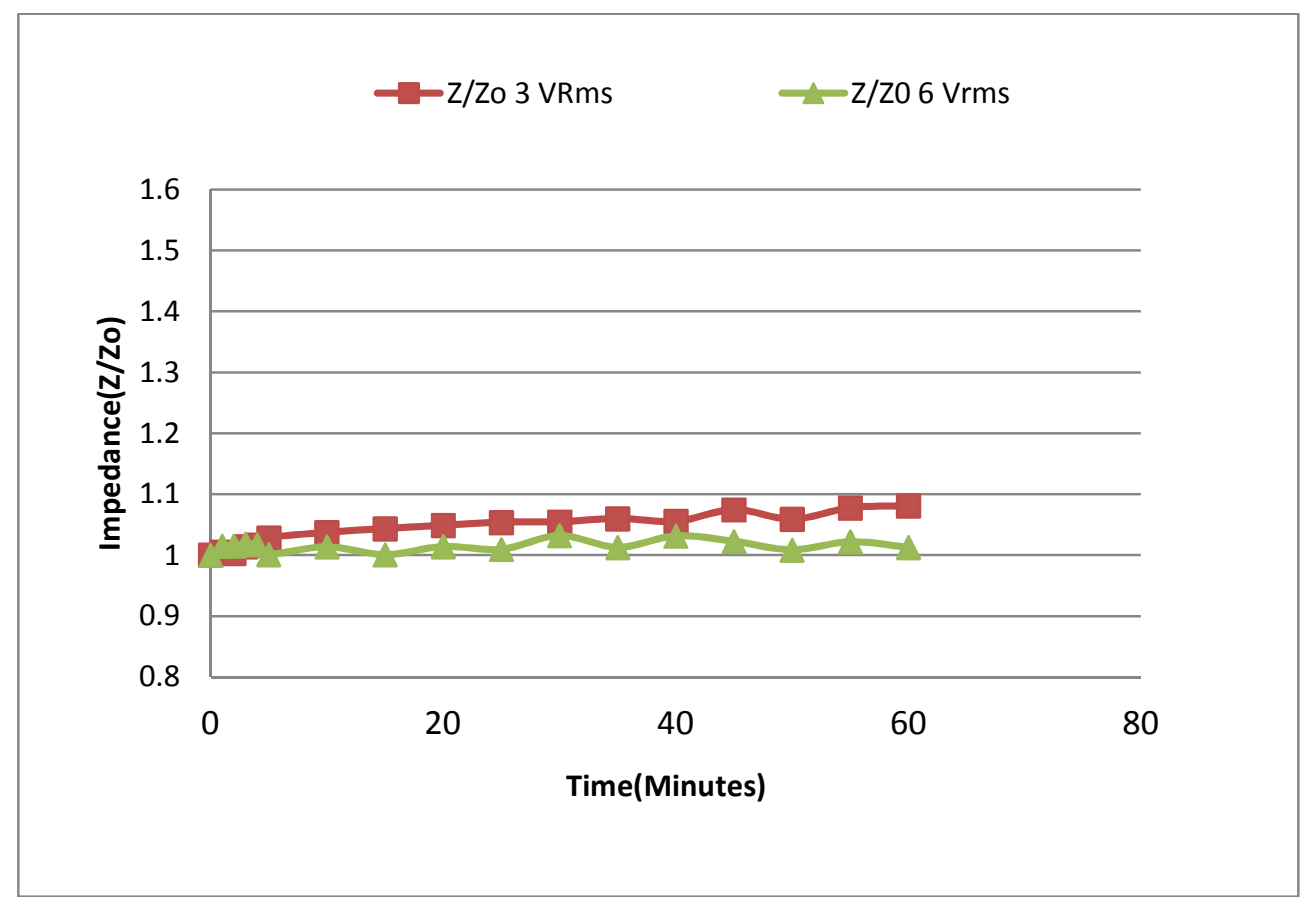

Fig. 7. Plot of Impedance vs. time for pristine PEDOT-PSS film making contact to $0.5 \mathrm{~mm}$ wide copper contact for shown rms voltages at $10 \mathrm{KHz}$ 


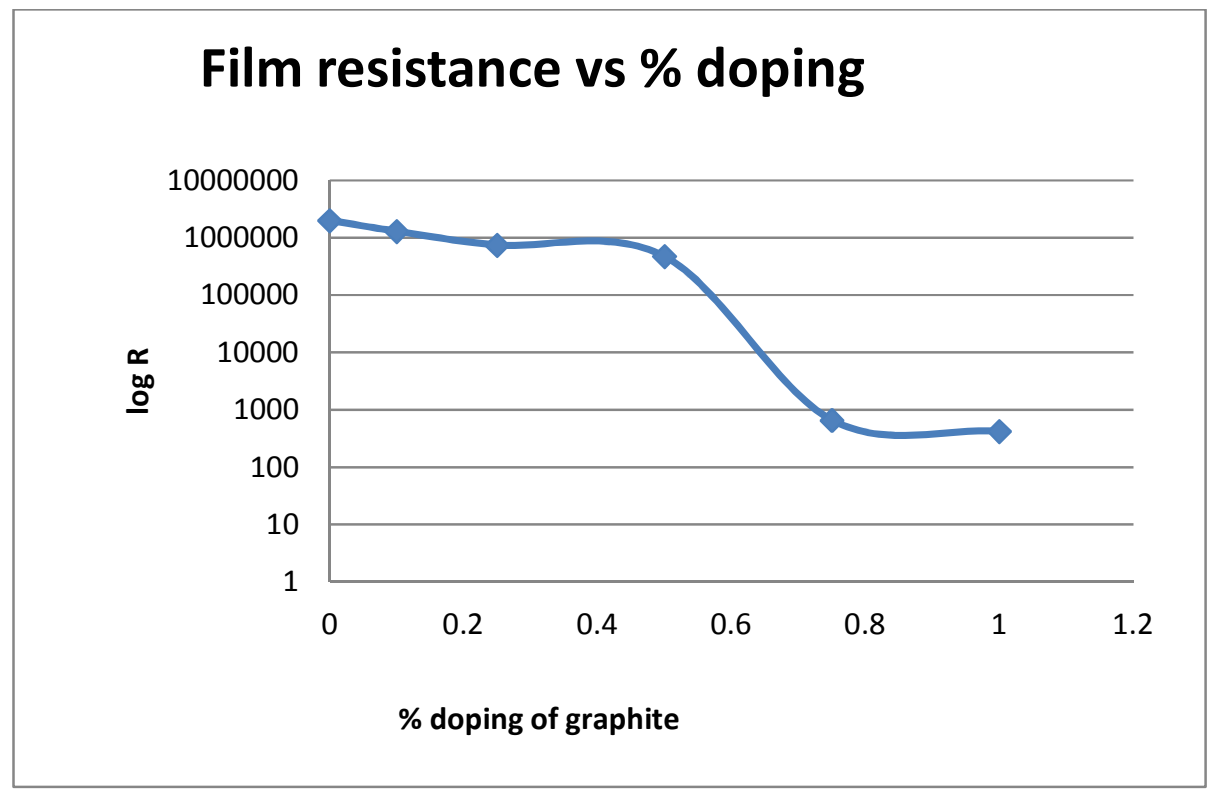

Fig. 8. Variation of film resistance with concentration of graphite into PEDOT-PSS

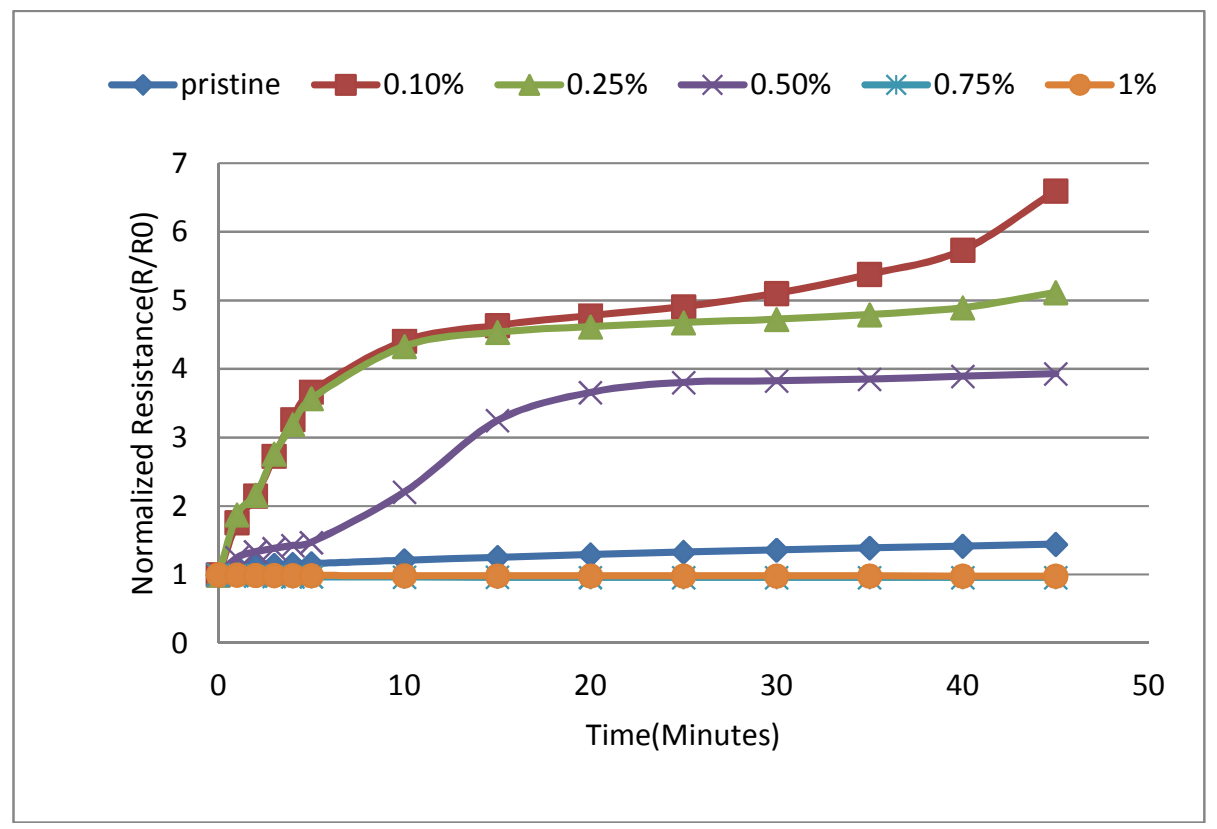

Fig. 9. Normalized resistance vs. time for PEDOT-PSS films dispersed with different concentrations of graphite powder

It may be observed that the extent of variation of film resistance with time is 1 to 7 , a remarkable increase, depending on the percentage of graphite dispersed. It may also be observed from Fig. 9 that the variation of resistances with time is 1.5 times for pristine PEDOT-PSS which increases suddenly to 7 times for $0.1 \%$ graphite powder dispersed film and then reduces as the dispersing concentration is increased and at $1 \%$ the resistance is nearly constant with time. Increased dispersion of graphite powder in PEDOT-PSS films nearly eliminates increase of resistance and stabilizes resistance; there is a sudden increase in the rate of resistance increase on low dispersion (0.1\%) which decreases on higher doping. The above 
observations can be explained by the invoking the interfacial diode where one diode is forward biased and another diode reverse biased.

\subsection{Impedance (Resistance) of PEDOT- PSS Film Dispersed with Graphite Powder with AC Excitation}

As discussed earlier AC excitation practically removes the effect of interfaces that lead to large change in the overall resistivity. So AC measurements are made on graphite dispersed films. The variation of normalized AC impedance with time for films dispersed with different concentrations of graphite is shown in Fig. 10. It may be observed that with $A C$ excitation the variation of film resistance (impedance) with time is very minimal and variation of resistance is very low compared to DC excitation.

\subsection{Discussion on the Cause of Resistance Increase after Application of DC Voltage}

First we can summarize the observations made till now:

1. Resistance of pristine PEDOT-PSS film increases with time on application of DC voltage.

2. The resistance measured consists of bulk resistance and the leakage resistance of an interfacial diode.
3. The interfacial diode dominates the increase in resistance as proved by $A C$ resistance.

4. AC excitation of the film bypasses the interfacial resistance.

5. Doping PEDOT-PSS with graphite reduces film resistance.

6. At $0.1 \%$ graphite doping resistance of doped films increases by a factor of 7(see Fig. 9) compared to 1.8 times for pristine films. However, at concentrations above $0.75 \%$ graphite the resistance variation is negligible.

Based on the above observations one can conclude that the variation of resistance is concentrated at the metal film interface.

Mrunal S. Mahajan et al. [12] have reported in their paper that the PEDOT-PSS film subjected to intense electric field of the order $2-5 \mathrm{KV} / \mathrm{cm}$ at $120^{\circ} \mathrm{C}$ for long time showed decrease of electrical conductivity over time in the plane of the electric field. If this is the possible reason for increase in resistance of PEDOT-PSS films how such high electric field is generated? If we look at the diode model at the metal PEDOT-PSS interface we observe a large voltage is dropped at the interfacial diode as the leakage resistance is $4-6$ times larger than the bulk resistance. The voltage drop in a reverse biased diode occurs in a very small width of its depletion region and hence the electric field in this region is intense compared to any other region of the film. An interfacial capacitance of $35 \mathrm{nF}$ was

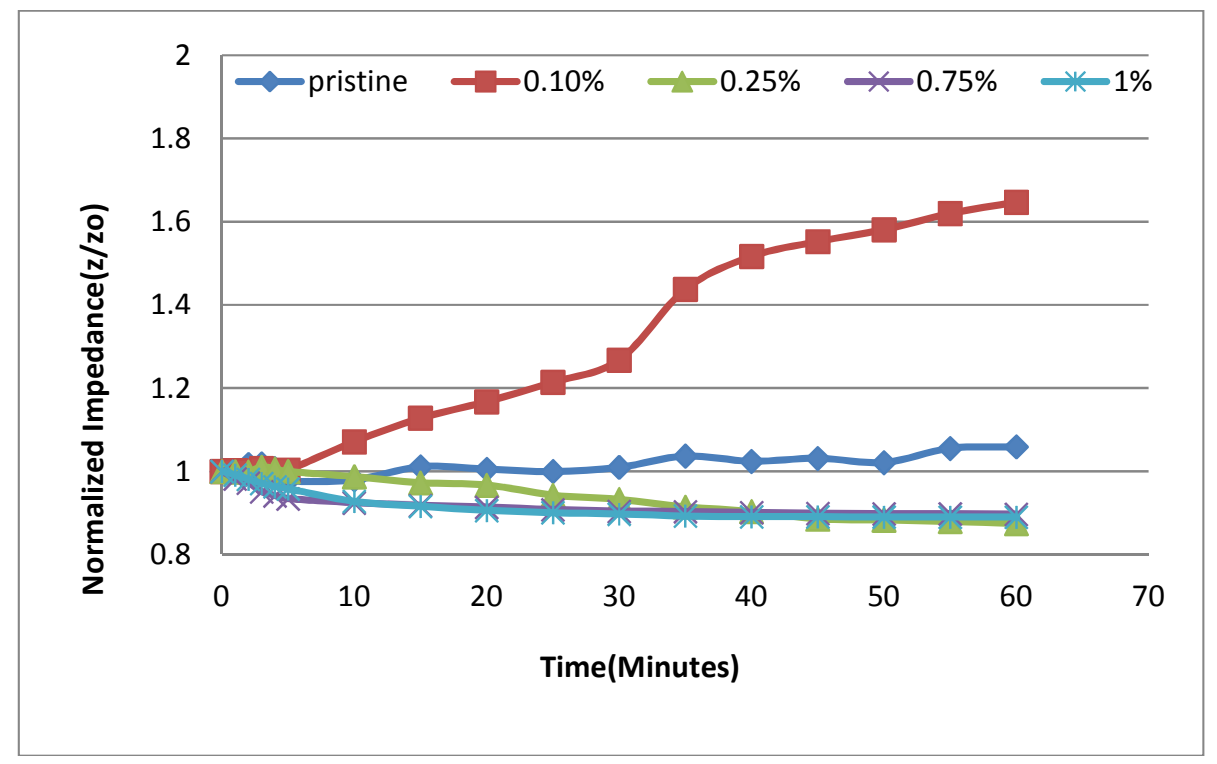

Fig. 10. Normalized impedance plot vs. time for different percentages of graphite doping 
measured using precision impedance analyzer, Agilent 4294 and found that the interfacial capacitance varies from about $17 \mathrm{nF}$ at low frequency to about 2 to $3 \mathrm{nF}$ for frequency above $100 \mathrm{KHz}$. For example if $3 \mathrm{~V}$ is dropped at a depletion width of $1 \mu \mathrm{m}$ the resulting electric field is $3^{*} 10^{6} \mathrm{~V} / \mathrm{m}$ which is large. This conclusion together with the observation that resistance increase is largely at the interface leads us to conclude that the variation of resistance is due to high electric field generated at the depletion region. When PEDOT-PSS is lightly dispersed with graphite the depletion layer thickness at the contact reduces as more current carriers are available. So, the electric field at the contact is higher consequently increasing the rate at which resistance increases. It may also be observed that with $0.1 \%$ doping of graphite the relative rise of resistance increases to 7 which decreases as the concentration of dopant is increased. So, when the film is doped the depletion layer thickness decreases producing higher electric field at the interface before the interface turns ohmic due to high tunneling which is evident at $1 \%$ doping. The reduction of variation of resistance between $0.1 \%$ doping to $1 \%$ doping is a region of complex nature where several competing phenomenon contribute to changing behavior of interface from rectifying to ohmic.

The above discussion explains two observations; increase in resistance with time and increase of rate of increase of resistance on low level dispersing, very low change of resistance with time at higher concentration of $1 \%$ graphite. This is consistent with the observations reported by Mrunal S. Mahajan et al. [5] that the conductivity of PEDOT-PSS film reduces on application of high electric field.

\subsection{Comparison of Resistance with DC and AC Excitation in Pristine PEDOT- PSS Films}

It is interesting to compare the effect of DC and $A C$ excitation on the same scale. Variation of film resistance with $D C(3 \mathrm{~V})$ and $A C$ (3Vrms) excitation is compared on the same scale and the resistances are normalized to their initial values as shown in Fig. 11.

It may be observed that $R_{A C}(Z)$ is constant with time which indicates that the interface resistance is bypassed by $A C$ input which measures the true bulk resistance of the film which is fairly constant with time due to low electric field.

The variation of resistance with time is an important information needed for making good strain measurements. The above discussion shows that the resistance of PEDOT-PSS films varies with time due to increase in electrical resistivity caused by high electric field at the reverse biased contact diode. Interfacial capacitive reactance bypasses the contact resistance at a suitable high frequency and allows more accurate time measurement of film bulk resistance when made by $A C$ excitation. It is also observed that making the interface ohmic by dispersing 1\% graphite will reverse the time variation of DC resistance. It may also be observed that the variation of pristine film resistance is negative for $1 \mathrm{~V}$ when the electric field at the interface is low. However at $3 \mathrm{~V}$ and above the electric field at the interface is large enough to cause increase in resistance. With these observations one can conclude that a large part of the variation of resistance in PEDOT-PSS films with time comes from the interface.

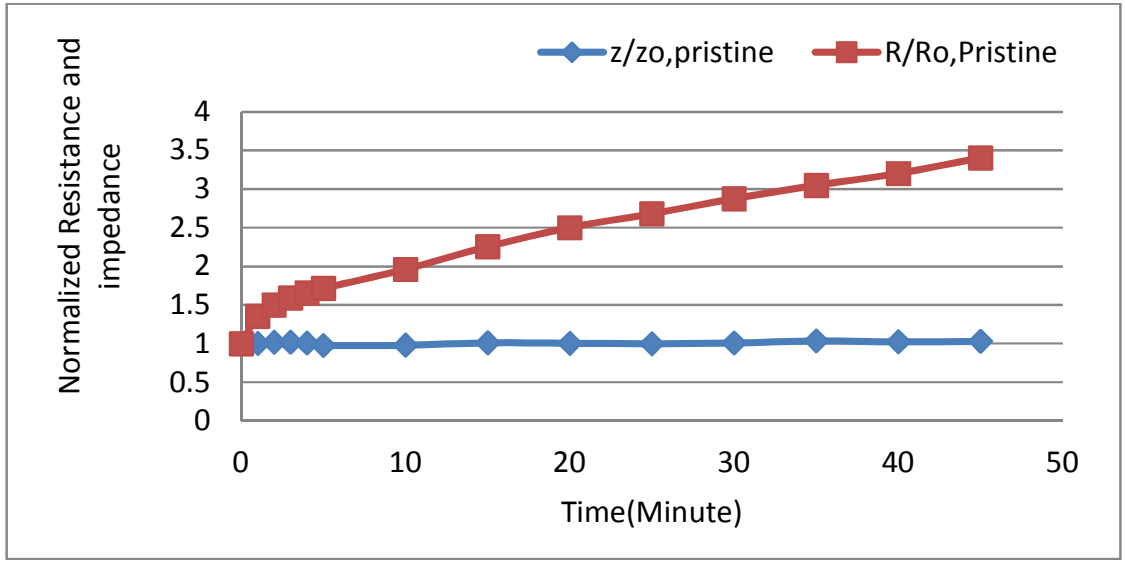

Fig. 11. Normalized resistance and Impedance plot for $6 \mathrm{~V} \mathrm{DC}$ and $6 \mathrm{Vrms}$ 
There is another aspect to be considered apart from high field at the interface is the concentration of heating at the interface. Fig. 12 shows the photograph of a PEDOT-PSS film formed by the method described earlier except the terminals are connected to a $5 \mathrm{~V}$ power supply during baking process.

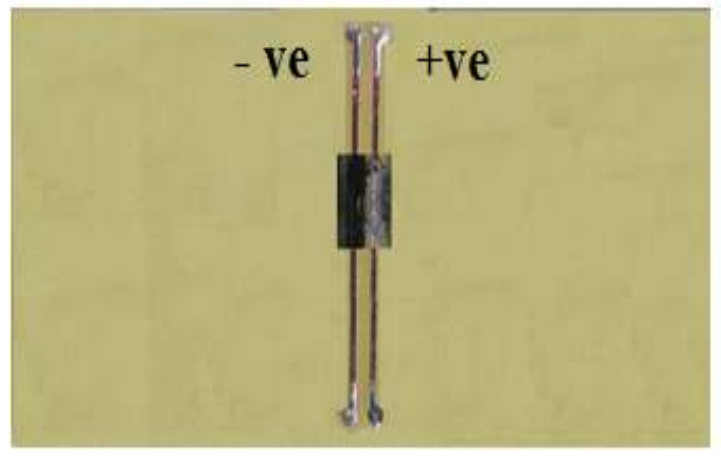

Fig. 12. Image of PEDOT-PSS film taken after film formation (DC potential was applied when the film is getting formed)

It is evident that the film is burnt near the +ve terminal while a good adherent film is formed at the -ve terminals. This observation is indeed a graphic proof of formation of diodes at the interface and concentration of heat at the +ve terminal. The burning has occurred at the reverse biased diode where most of the dissipation occurs and locally the temperature is high. This causes the water to evaporate faster causing blisters locally. This is a good proof that heating is intense at the interface and locally the temperature is high.

\section{CONCLUSION}

The study shows that the resistance of PEDOTPSS films increases with time between two metal contacts (contrary to negative temperature coefficient reported). The increase is attributable to high electric field in the interfacial region of the PEDOT-PSS/metal contact with consequent slow reduction in the interfacial conductivity. AC excitation bypasses the interfacial resistance and gives bulk resistance.

It has conclusively been shown that increase in resistance is largely an interfacial phenomenon. The variation of resistance with time is important information needed for making good strain measurements.

\section{ACKNOWLEDGEMENTS}

We thank management of $\mathrm{B} N \mathrm{~N}$ Institute of Technology, Bangalore, for permission and support in carrying out the work.

\section{COMPETING INTERESTS}

Authors have declared that no competing interests exist.

\section{REFERENCES}

1. Kim WH, Mäkinen AJ, Nikolov $\mathrm{N}$, Shashidhar R, Kim H, Kafafi ZH. Molecular organic light-emitting diodes using highly conducting polymers as anodes. Applied Physics Letters. 2002;80(20): 3844-6.

2. Ramona Mateiu, Michael Lillemose, Thomas Steen Hansen, Anja Boisen, Oliver Geschke. Reliability of Poly3, 4ethylenedioxythiophene strain gauge. Microelectronics Engineering. 2007;84: 1270-1273.

3. Giuseppe Latessa. A glove-based system equipped with home-made piezoresistive bend sensors; 2010.

Available:http://art.torvergata.it/handle/210 $\underline{8 / 1388}$

4. Schweizer Thomas Martin. Electrical characterization and investigation of the piezoresistive effect of PEDOT: PSS thin films. Diss. Georgia Institute of Technology; 2005.

5. Ulrich Lange, Vladimir M. Mirsky, Separated analysis of bulk and contact resistance of conducting polymers: Comparison of simultaneous two- and four point measurements with impedance measurements, short communication

6. Alborghetti S, Coey JMD, Stamenov P. Dependence of charge carrier injection on the interface energy barrier in short channel polymeric field effect transistors. Appl. Phys. Let. 2012;100:143301.

DOI: $10.1063 / 1.3701271$

7. Bindu S, Anil Kumar R, Suresh MS. Development of technique for making Ohmic contacts to PEDOT-PSS films. Proceedings of International Conference on VLSI, Communication, Advanced Devices, Signals \& Systems and Networking (VCASAN-2013). Springer India; 2013. 
8. Bindu S, Suresh MS. Measurement of bulk resistance of conducting polymer films in presence of rectifying contacts. International Journal of Scientific and Research Publications. 2014;19.

9. Bindu S, Suresh MS. Ohmic contact formation to PEDOT-PSS films using graphite-clay. British Journal of Applied Science \& Technology. 2015;6(4):342.

10. Forlani F, Prudenziati M. Electrical conduction by percolation in thick film resistors. Electrocomponent Science and Technology. 1976;3:77-83.
11. Gulmurza Abdurakhmanov. On the conduction mechanism of silicate glass doped by oxide compounds of ruthenium (Thick film resistors)-Diffusion and percolation levels. World Journal of Condensed Matter Physics. 2011;1: 19-23.

DOI: 10.4236/wjcmp.2011.12004

12. Mahajan Mrunal $S$. Changes in in-plane electrical conductivity of PEDOT: PSS thin films due to electric field induced dipolar reorientation. RSC Advances. 2015;5(105): 86393-86401.

(0) 2017 Bindu and Suresh; This is an Open Access article distributed under the terms of the Creative Commons Attribution License (http://creativecommons.org/licenses/by/4.0), which permits unrestricted use, distribution, and reproduction in any medium, provided the original work is properly cited.

Peer-review history:

The peer review history for this paper can be accessed here: http://sciencedomain.org/review-history/18997 